\title{
A Brick-Sorting LEGO Robot
}

\author{
Arturo Pérez and Michael R. W. Dawson \\ Department of Psychology, University of Alberta
}

\begin{abstract}
Arturo Pérez is a senior undergraduate psychology student at Universidad Diego Por-tales in Santiago, Chile. In the Fall term of 2012, Arturo spent 3 months at the Universi-ty of Alberta, hosted by Dr. Michael Dawson and the Biological Computation Project (BCP). The general goal of his visit was to establish collaborative ties between this $U$ of A laboratory and the Centro de Estudios de la Argumentación y el Razonamiento (CEAR) at UDP. A more specific purpose was to explore the BCP's approach to using simple robots to explore basic ideas in embodied cognitive science. Arturo's explora-tions involved creating, programming, and testing a new robot designed to sort ele-ments in an arena. The purpose of the current paper is to report on Arturo's robotics research at the BCP.
\end{abstract}

\section{PURPOSE}

Behavior-based robots are simple machines built from a number of basic sense-act reflexes (Brooks, 1999; Sharkey, 1997). They do not create and use complex represen-tations of their external world to plan and guide their behavior. Instead, their external world serves as its own representation; complex behaviors emerge because the robot's sensors immediately detect properties in the world, properties that trigger adaptive responses tuned to environmental stimuli.

Research on behavior-based robots is often biomimetic (Sharkey, 2006; Webb \& Consi, 2001). Researchers, seeing the complex behavior of simple organisms in the natural world, explore the possibility of achieving similar behavior in simple robots by exploiting sense-act processing. For instance, robotic models of cricket phonotaxis, in which female crickets orient to particular cricket songs to choose a mate, have resulted in extraordinarily simple and plausible new theories of this phenomenon (Webb, 1996; Webb \& Scutt, 2000).

One natural behavior that has inspired a great deal of robotic research is the brood sorting of ants (Franks \& Sendova-Franks, 1992; Sendova-Franks, Scholes, Franks, \& Melhuish, 2004). Many species of ants establish their colony in what is in essence a two-dimensional space, for instance, spread out in a plane that is covered by a large flat rock. As the eggs laid by the queen ant develop into larvae, they grow in size and require greater care. Workers in the colony facilitate this care by moving larger eggs and larvae towards the perimeter of the nest. Thus, if one quickly removed the rock covering the colony, they would see its brood organized spatially in an approximately circular arrangement; as one moved in any direction from the center of the brood one would note an increase in the size of the arranged eggs and larvae. Brood sorting has inspired several studies that attempt to achieve sorting behavior in behavior-based robots (Deneubourg et al., 1991; Holland \& Melhuish, 1999; Melhuish, Sendova-Franks, Scholes, Horsfield, \& Welsby, 2006; Scholes, Wilson, Sendova-Franks, \& Melhuish, 2004; Wilson, Melhuish, Sendova-Franks, \& Scholes, 2004).
One example of such a device is a simple Lego NXT robot named the Lemming (Dawson, Dupuis, \& Wilson, 2010). The Lemming moves about a rectangular arena on wheels driven by two separate motors. It explores around its environment when its motors rotate at different speeds. An ultrasonic sensor mounted on its top detects obstacles, and causes the robot to steer away from them. An assortment of Lego bricks is scattered throughout the Lemming's environment. The robot steers towards bricks detected by a lower ultrasonic sensor. When captured by the robot's 'plow', a light sensor determines whether a brick is white or black. The robot deposits white bricks close to walls, and deposits black bricks close to other bricks. All of these behaviors are accomplished with a simple sense-act hierarchy of reflexes called a subsumption architecture (Brooks, 1991; Brooks \& Flynn, 1989; Brooks, 1989). The Lemming produces some surprising and unexpected sorting behaviors, and demonstrates swarm intelligence, because sorting efficiency increases exponentially as more Lemmings are added to the arena.

The sorting behavior of the Lemming is interesting and successful. However, some of its abilities are in obvious need of improvement. For example, the lower ultrasonic does not provide very efficient steering towards to-be-sorted bricks. As a result, it takes a single Lemming over an hour to sort the bricks in its testing arena (Dawson et al., 2010).

The purpose of the current research was to develop a brick-sorting robot using different sensors, which require a different subsumption architecture. Initially, the motivation for the research was to improve upon the performance of the original Lemming. However, once the new robot was constructed and programmed, our interest turned to exploring new sorting behaviors that it produced, behaviors that were quite different from those exhibited by the original robot.

This paper proceeds as follows: first, we briefly describe the embodiment of the ro-bot. Second, we define the basic behaviors that make up its subsumption architecture. Third, we illustrate the interesting sorting behaviors that it produces, and which depend upon the environment in which the robot is situated. We conclude by briefly discussing the implications of our robot. 


\section{THE EMBODIMENT OF 'ARTURITO’}

We named the new robot Arturito, inspired by the first name of its inventor and acknowledging its vague resemblance to the Star Wars robot R2-D2. Arturito is approximately 8 inches high, supported by a pair of wheels and by a plow mounted on its front. The body of Arturito is a Lego NXT brick. On the top of the robot is an ultrasonic sensor used to mediate obstacle avoidance. The robot's plow enables brick capture and pushing. The shape of the plow is critical: when the robot spins to avoid an obstacle, it leaves a carried brick behind. The plow also supports light sensors for detecting and steering towards bricks, as well as for measuring the color of bricks after their capture. Figure 1 illustrates Arturito's embodiment.

\section{THE CORE BEHAVIORS OF ARTURITO}

Arturito's behavior is created by employing a subsumption architecture that defines a hierarchical arrangement of simple sense-act reflexes. This subsumption architecture was programmed in the NXC language using the Bricx programming environment, which is freely available on the internet.

The most basic level of Arturito causes its two motors to run at a base speed, driving the robot forward.

The second level of its architecture uses the ultrasonic sensor to avoid obstacles. When the ultrasonic detects that an obstacle is closer than a safe threshold, motor

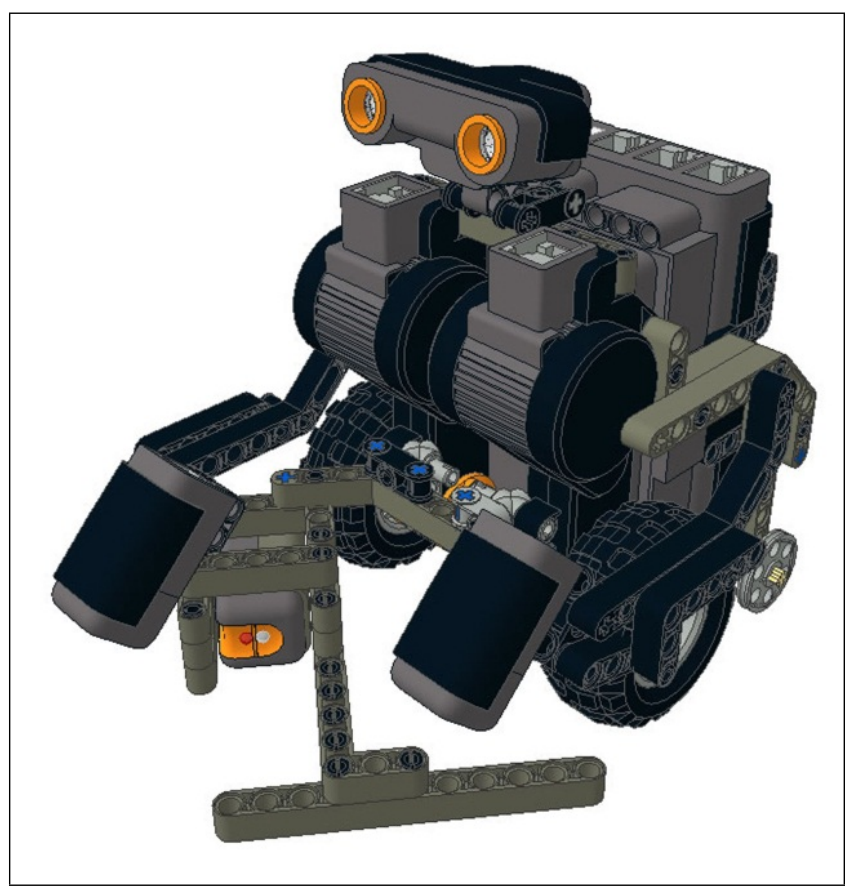

FIG. 1. Arturito, the brick-sorting Lego NXT robot. directions are manipulated to turn Arturito away.

The third level of Arturito's architecture uses two lower light sensors to alter the speed of the two motors. The steering mechanism is a variation of a common robot, Braitenberg Vehicle 2 (Braitenberg, 1984; Dawson et al., 2010). Originally, we hoped that when the light sensors detected reflections, the robot would turn towards any brick. Indeed, this approach was one of the more innovative aspects of Arturito, because we had never explored the steering capability of light sensors when the desired targets were not themselves lights. However, and as is discussed below, we discovered that this level produced sharp steering towards white bricks, but steered Arturito away from black bricks. Thus one behavior that emerged from level 3 was a taste for white bricks, and an aversion to black bricks.

The final level of Arturito's subsumption architecture was invoked when a brick was captured in the robot's plow. The color of the brick altered Arturito's obstacle threshold. The threshold decreased if the brick was white, with the intent of having white bricks deposited near arena walls. In contrast, the threshold increased if the brick was black, with the intent of having black bricks deposited further from arena walls. This level also inhibited Arturito's brick-seeking behavior, with the intent of having the robot transport a brick without simultaneously seeking another.

\section{ARTURITO'S SORTING BEHAVIOR}

We studied Arturito's sorting behavior in the same arena that housed the original lemming. The arena was a small lab room, approximately $8^{\prime}$ x $6^{\prime}$; a camera mounted in the ceiling recorded brick sorting. We began by placing a regular arrangement of Lego bricks on the linoleum floor of the arena in a typical study. Figure 2 illustrates an example initial configuration of bricks. We randomly placed the robot in the room, activated it, and closed the door as we left the machine to run on its own. We conducted a number of simple studies as is detailed below. We were interested in Arturito's behavior when it encountered bricks that were all the same color, as well as various mixtures and ar-rangements of different colored bricks. The text below provides brief descriptions of our results; a video that illustrates the robot and its sorting behavior is also available at the following link: http://www. youtube.com/watch?v= jTRBgAuhFaY

\section{A. White Brick Sorting}

We began by observing how the robot would behave in a room with moderate light that only contained white bricks. The performance of the robot in these conditions indicated that our embodiment and programming 


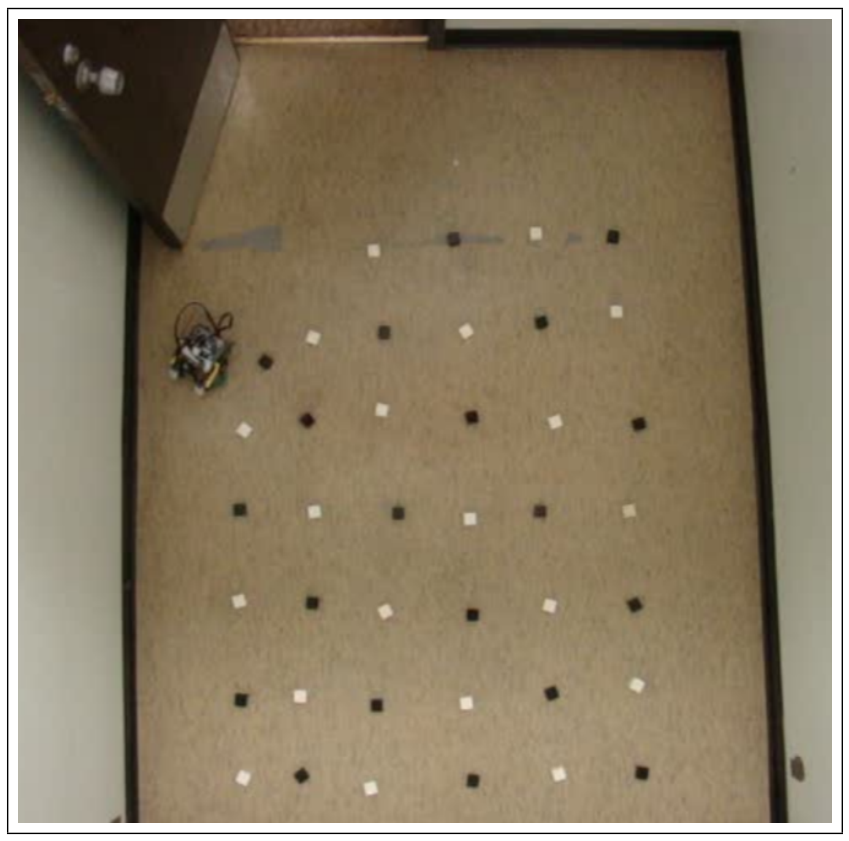

FIG. 2. An example starting state for robot sorting.

were very successful. First, the robot turned to a detected brick as if it was looking for it; this behavior was far more effective than that observed in the Lemming. After scooping up a brick in its plow, it typically drove itself to a nearby wall, ignoring the presence of other bricks along its way. Finally, it turned away from the wall, depositing the brick that it carried. Second, brick sorting behavior was much faster than that seen in the Lemming. After only about 10-15 minutes, Arturito had pushed all of the white bricks near a wall, leaving the centre of the arena empty, as shown in Figure 3.

In some instances, Arturito's behavior was surprising. Sometimes when the robot had a brick in its plow it still steered towards others. In these situations, the second brick stayed just in front of the sensor, and Arturito moved around in spirals. However, eventually another brick would attract the robot, steering it in a direction that caused it to deposit its (multiple) bricks. This returned Arturito's behavior to normal.

\section{B. Black Brick Sorting and a Taste for White Bricks}

In a second study, Arturito's arena contained only black bricks. We expected in this case that there would merely a quantitative difference between this study and the one described above. That is, we expected that Level 3 would cause the black bricks to be arranged in a similar fashion to the white bricks, with the exception that they would be further from the walls.

Instead, we surprisingly found that Arturito steered away from the black bricks. The black bricks reflected light back to the light sensors in such a way that the re-

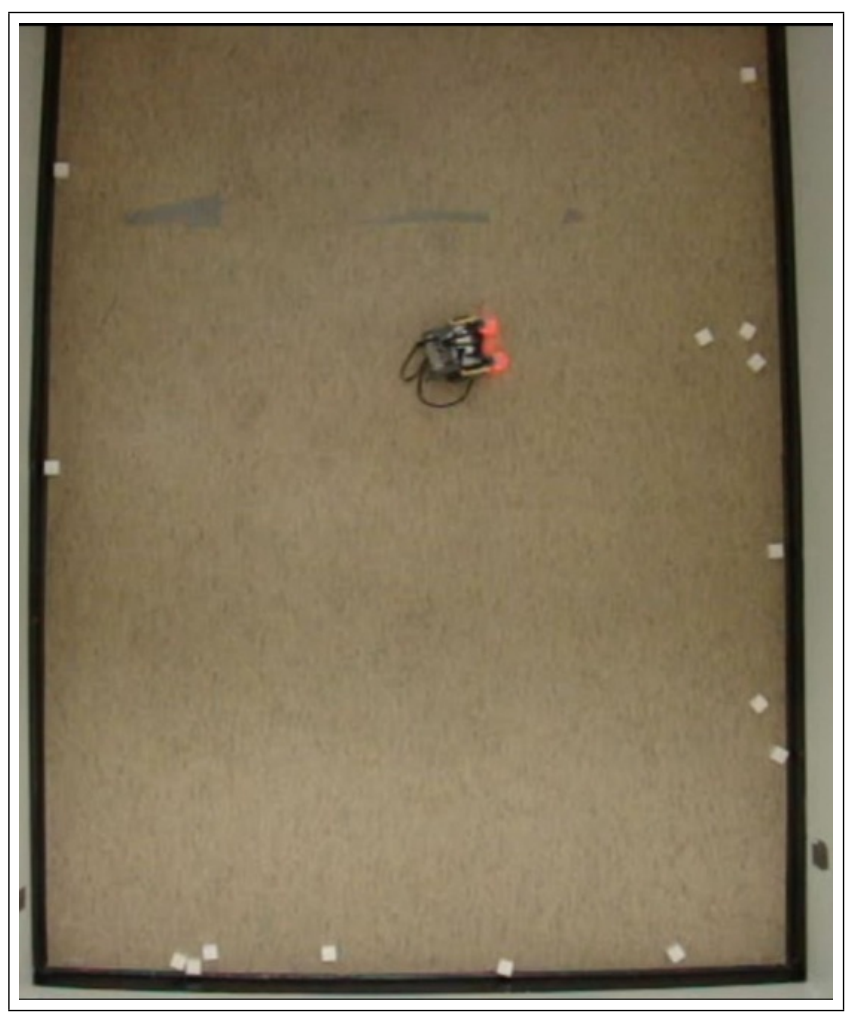

FIG. 3. Arturito clears white bricks from the arena's center.

sulting motor behaviors caused aversion instead of attraction. In the spirit of Braitenberg (1984), we might say has a "taste", it likes white bricks and dislikes black bricks. Note that we obtained this selective behavior "for free', in the sense that we did not explicitly write different code to cause the machine to steer differently in response to different colored bricks. Instead, Arturito's "taste" emerges from an interaction between its Level 2 architecture and the way in which light reflects from different colored bricks.

The result of all of this is that in contrast to the previous study, Arturito leaves the center of the arena occupied by an expanse of black bricks. A typical final configuration is provided in Figure 4.

\section{Sorting Black and White Bricks}

Many of our studies examined Arturito's performance when placed in an arena that contained both white and black bricks. In this kind of situation, the robot's behavior was quite interesting because of its tendency to steer towards white bricks and to steer away from black bricks, combined with its obstacle-avoiding threshold being determined by the color of brick that was in the robot's plow. We were able to produce a wide variety of sorting behaviors by experimenting with the relative proportions of white and black bricks, as well as with their initial arrangement. 


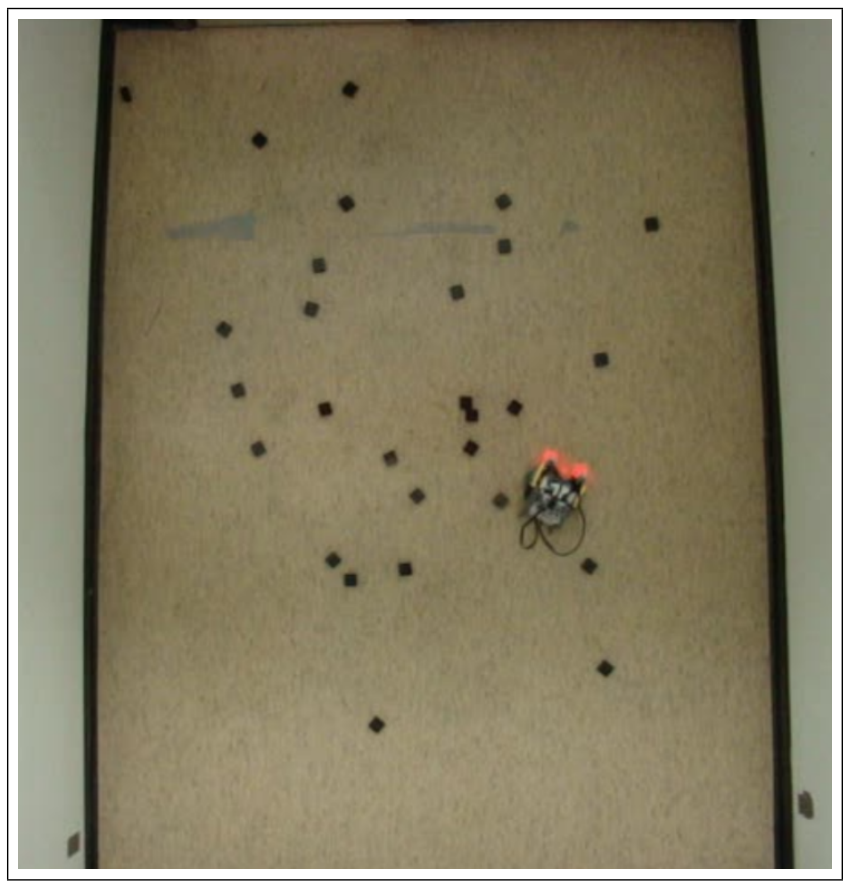

FIG. 4. Arturito leaves black bricks in the middle of the arena.

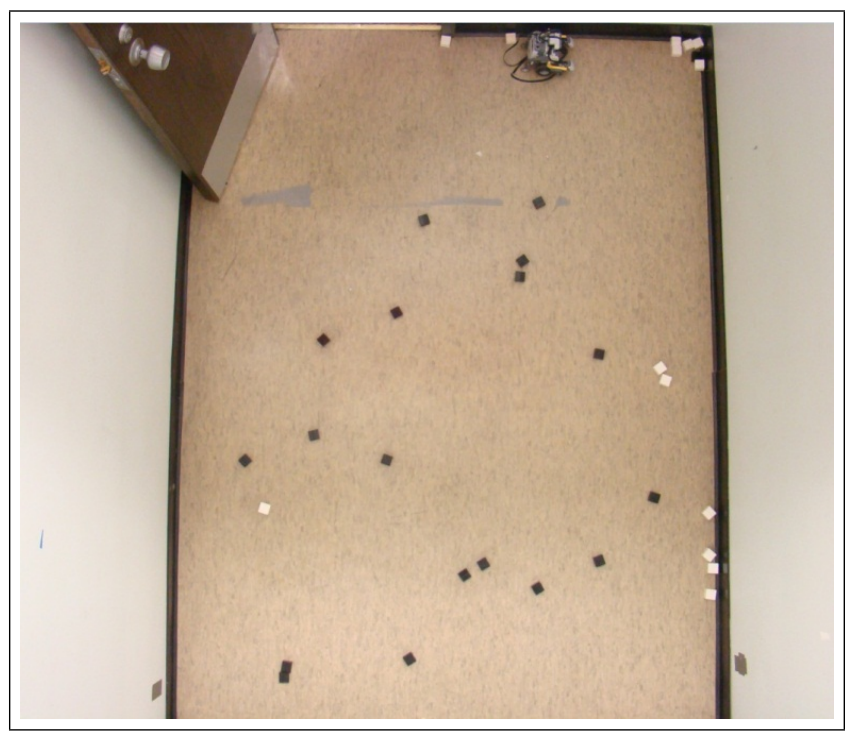

FIG. 5. Arturito has sorted the configuration of bricks from Figure 2.

When the robot was confronted with a regular arrangement of black and white bricks in a regular arrangement (Figure 2), it sorted bricks in a fashion that was similar to, but more efficient than, the Lemming. For example, the sorted pattern presented in Figure 5 required only 21 minutes, in comparison to the $70+\mathrm{min}$ utes that the lemming would require to produce a similar result.

Interestingly, Figure 5 indicates that Arturito moved more black bricks out of the arena than was the case when only black bricks were present (Figure 4). This reflects the influence of the white bricks in the configuration, which attracted Arturito - and caused it to pick up a black brick as it approached the white one! As well, the pattern of Figure 5 is analogous to the annular arrangement that is sought in models of brood sorting (Wilson et al., 2004). This raises questions for further research. How much of annular brood sorting is due to a preference for one type of brood item over another? To what extent is the annular arrangement of sorted items dependent upon the size and shape of the environment?

In other studies, we observed how the robot would behave with different proportions of bricks of different color. In one, we selected a 3:1 proportion of black to white bricks, placing the white bricks in the center and the black ones around them, making some kind of a surrounding 'membrane'. Generally, the robot was repelled by this 'membrane', but eventually broke through it and started the sorting of white bricks. We also noted that black bricks sometimes made it harder for the robot to sort particular bricks by "hiding" them, making almost impossible for the robot to sort it because every time the robot came near it was repelled by the black bricks. Finally, particular arrangements of bricks that emerged in the middle of sorting could alter Arturito's trajectory through the arena. For example, if a straight line of black bricks emerged, then the robot would travel parallel to this line. Many of these intriguing behaviors can be seen in the video whose link was provided earlier.

\section{IMPLICATIONS AND CONCLUSION}

Our observations of Arturito suggest that we successfully achieved the initial goals of the robot project. By altering the embodiment of the Lemming, and by revising the subsumption architecture, we developed a robot that achieved brick sorting far more efficiently. The robot's unexpected preference for one color of brick over another led to some interesting sorting patterns; the Lemming would not produce the annular ar-rangement in Figure 5.

Arturito's seeking of white bricks, and avoiding of black ones, suggests future studies that explore environmental influences to a much greater degree than is reported here, or than was used to study the Lemming. Of great interest is the extent to which the ini-tial configuration of differently colored bricks affects the final pattern of sorting. In particular, some initial arrangements might either facilitate or hurt sorting; might they also determine the final arrangement of sorted items? As well, we expect that the size and the shape of the robot's arena will also have a profound influence on the final sorting result.

One aspect studied in more detail with the Lemming was the emergence of collective intelligence. Increasing the number of Lemmings from 1 to 2 to 3 produced an 
exponential increase in sorting efficiency, which is a hallmark of collective intelligence (Sugawara \& Sano, 1997; Sulis, 1997). When two Arturitos work together, sorting behavior is very quick. However, whether this increase is exponential or not requires studying the behavior of a collective of 3 or more robots, which we have not done to date.

One of the interesting implications of this project is the extent to which sorting behavior is affected by the arrangement of different elements that elicit different behaviors (approach or avoidance). This raises the obvious question about whether brood sorting by ants is governed by similar principles. The biomimetic nature of behavior-based robotics clearly is bidirectional, as robot behavior can raise questions to be studied using biological agents in their natural setting (Webb, 2000).

\section{REFERENCES}

Braitenberg, V. (1984). Vehicles: Explorations In Synthetic Psychology. Cambridge, MA: MIT Press.

Brooks, R. (1991). Intelligence without representation. Artificial Intelligence, 47, 139-159.

Brooks, R., \& Flynn, A. M. (1989). Fast, cheap and out of control: A robot invasion of the solar system. Journal of The British Interplanetary Society, 42, 478-485.

Brooks, R. A. (1989). A robot that walks; emergent behaviours from a carefully evolved network. Neural Computation, 1, 253-262.

Brooks, R. A. (1999). Cambrian Intelligence: The Early History Of The New AI. Cambridge, MA: MIT Press.

Dawson, M. R. W., Dupuis, B., \& Wilson, M. (2010). From Bricks To Brains: The Embodied Cognitive Science Of LEGO Robots. Edmonton, AB: Athabasca University Press.

Deneubourg, J. L., Goss, S., Franks, N., Sendova-Franks, A., Detrain, C., \& Chretien, L. (1991). The dynamics of collective sorting robot-like ants and ant-like robots, Proceedings of the first international conference on simulation of adaptive behavior (From animals to animats) (pp. 356-363). Paris, France: MIT Press.

Franks, N. R., \& Sendova-Franks, A. B. (1992). Brood sorting by ants: Distributing the workload over the work surface. Behavioral Ecology and Sociobiology, 30(2), 109-123.

Holland, O., \& Melhuish, C. (1999). Stigmergy, selforganization, and sorting in collective robotics. Artificial Life, 5, 173-202.

Melhuish, C., Sendova-Franks, A. B., Scholes, S., Horsfield, I., \& Welsby, F. (2006). Ant-inspired sorting by robots: the importance of initial clustering. Journal of the Royal Society Interface, 3(7), 235-242.

Scholes, S., Wilson, M., Sendova-Franks, A. B., \& Melhuish, C. (2004). Comparisons in evolution and engineering: The collective intelligence of sorting. Adaptive Behavior, 12(3-4), 147-159.

Sendova-Franks, A. B., Scholes, S. R., Franks, N. R., \& Melhuish, C. (2004). Brood sorting by ants: two phases and differential diffusion. Animal Behaviour, 68, 10951106.

Sharkey, A. J. C. (2006). Robots, insects and swarm intelligence. Artificial Intelligence Review, 26(4), 255-268.

Sharkey, N. E. (1997). The new wave in robot learning. Robotics and Autonomous Systems, 22(3-4), 179-185.

Sugawara, K., \& Sano, M. (1997). Cooperative acceleration of task performance: Foraging behavior of interacting multi-robots system. Physica D, 100(3-4), 343354.

Sulis, W. (1997). Fundamental concepts of collective intelligence. Nonlinear Dynamics, Psychology, and Life Sciences, 1, 35-53.

Webb, B. (1996). A cricket robot. Scientific American, 275, 94-99.

Webb, B. (2000). What does robotics offer animal behaviour? Animal Behaviour, 60, 545-558.

Webb, B., \& Consi, T. R. (2001). Biorobotics : methods and applications. Menlo Park, CA: AAAI Press/MIT Press.

Webb, B., \& Scutt, T. (2000). A simple latency-dependent spiking-neuron model of cricket phonotaxis. Biological Cybernetics, 82(3), 247-269.

Wilson, M., Melhuish, C., Sendova-Franks, A. B., \& Scholes, S. (2004). Algorithms for building annular structures with minimalist robots inspired by brood sorting in ant colonies. Autonomous Robots, 17(2-3), 115-136. 